TRANSLATED PAPER

\title{
Observation of traveling wave with laser tomography
}

\author{
Yusuke Ikeda*, Naoki Okamoto, Tadashi Konishi, Yasuhiro Oikawa, \\ Yasuhiro Tokita and Yoshio Yamasaki \\ Waseda University, 3-4-1 Ohkubo, Shinjuku-ku, Tokyo, 169-8555 Japan
}

(Received 28 March 2016, Accepted for publication 7 April 2016)

\begin{abstract}
In this study, we observe traveling sound waves with a laser and computed tomography. In our previous research, we observed steady-state sound fields using a sine wave signal. However, transient sound fields observation is necessary in order to observe more complicated sound fields that need to be separated into direct and reflected sounds. Therefore, in this study, we use a traveling sound wave (pulse sound wave). For fundamental experiments, we observed projections of a traveling pulse sound wave generated by a 2-way loudspeaker and a flat-panel loudspeaker with a laser. For transit sound fields, we used the sound field projections obtained with a laser to observe that a flat sound wave generated by a flat-panel loudspeaker is reflected by a sound-reflecting board. Then, we reconstructed the sound field information on the transition of a pulse sound wave generated by a 2 -way loudspeaker, by computed tomography.
\end{abstract}

Keywords: Laser Doppler vibrometer, Computed tomography, Pulse sound wave, Sound-reflecting board

PACS number: 43.58.Fm [doi:10.1250/ast.37.231]

\section{INTRODUCTION}

To understand sound fields, in previous researches, we carried out observations by various methods such as a closelylocated 4-point microphone method [1,2]. In recent years, we have proposed a method of sound field observation using a laser interferometer [3,4]. In addition, we reconstructed the distribution of sound pressures by applying computed tomography (CT) to sound field projections obtained using a scanning laser interferometer [5].

The characteristics of a sound field observation method with a laser are that the sound field information in a broad area is simultaneously observed, and the instruments do not need to be placed inside the observed sound fields.

The measurement techniques using a laser and CT have been applied in various research fields: visualization and temperature or optical index distribution measurement of flows for flames and supersonic jets, for example [6-8]. The previous researches for sound measurement with a laser have focused on the high power ultrasound [9]. The sound measurement methods with a laser have been

*e-mail: yusuke.ikeda@aoni.waseda.jp

The original paper (in Japanese) is published in the Journal of the Acoustical Society of Japan, 64(3), pp. 142-149 (2008). studied: the measurement of supersonic sound with a laser reflector (rigid body), which can be assumed not to vibrate, and the measurement of a directional sound field with a mirror [10,11]. We observed various sound fields and conducted experiments by applying a laser and CT to audible sound $[5,12,13]$. In this study, we use a laser Doppler vibrometer and a rigid body as a laser measurement system.

In our previous researches, we observed steady-state sound fields generated by loudspeakers. The measurement signal was a continuous sine signal; we observed sound fields of the specified frequency [5]. However, to observe complicated sound fields such as reflections of sound, it is necessary to observe a transit sound field.

In this study, we observe traveling sound waves with a laser interferometer, using a pulse sound wave as a measurement signal. For fundamental experiments, we observed laser projections of traveling sounds generated by two types of loudspeaker: a 2-way loudspeaker and flatpanel loudspeakers. We also conducted an experiment with laser projection to observe a transit sound field; a flat sound wave generated by flat-panel loudspeakers is reflected by a sound reflector. In addition, we obtain more information on the sound field through the reconstruction of sound information at each point by applying the $\mathrm{CT}$ to the projections. 


\section{OBSERVATION OF TWO-DIMENSIONAL PROJECTION OF PULSE SOUND WAVE USING LASER INTERFEROMETER}

\subsection{Observation of Sound Wave Using Laser Inter- ferometer}

Sound is a change in the air density. Because the refractive index changes with air density, the light path length of a laser that passes through a sound field is changed by a sound wave. The interferometer uses the interference of two light waves that propagate through different paths to measure the change in the laser light path length. That is, the measurement of the light path length change caused by a sound field is obtained from the interference of a light wave that passes and one that does not pass through the sound field.

We use a scanning laser Doppler vibrometer (LDV) as a laser interferometer. The LDV irradiates a vibrating object with one laser; the reflected laser interferes with another laser. Then, the LDV measures the vibration speed of the object from the change in its interference fringe.

When we use a rigid object, which is assumed not to vibrate, we measure the changes in the refractive index caused by sound [10]. The change in the light path length, $d \phi / d t$, measured with the LDV is represented by

$$
\frac{d \phi}{d t}=\int_{C} \frac{\partial n(s, t)}{\partial t} d s
$$

where $n(s, t)$ at coordinate point $s$ on laser path $C$ is the difference in the refractive index between the atmosphere and the sound field.

We measure sound field information along multiple laser paths by scanning with a laser. In the following section, we describe the method of time-based sound field observation using synchronized sound field projections.

\subsection{Method of Observation of Pulse Sound Wave}

In our previous research, we observed a steady-state sound wave. In this study, we use a pulse signal as a measurement signal to observe a transit sound field. Figure 1 shows the measurement system [14].

The sound field to be measured is a reproducible sound field generated by loudspeakers. When we scan a sound field with a scanning LDV, it is necessary to reproduce the sound field for each scan. Therefore, as shown in Fig. 1, the trigger signal synchronized with a pulse signal that is the input signal of the loudspeaker is sent to the LDV. The trigger signal marks the start time of measurement at each point of the projection. The output of LDV is recorded after the start time. The span of measurement time is long enough to observe the pulse sound wave to be measured.

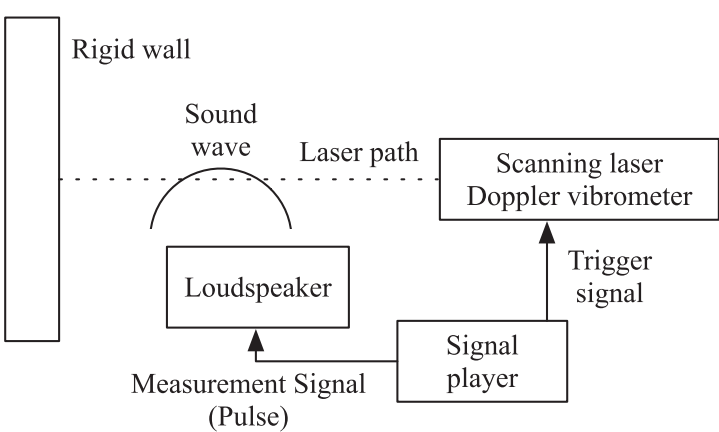

Fig. 1 Measurement system.

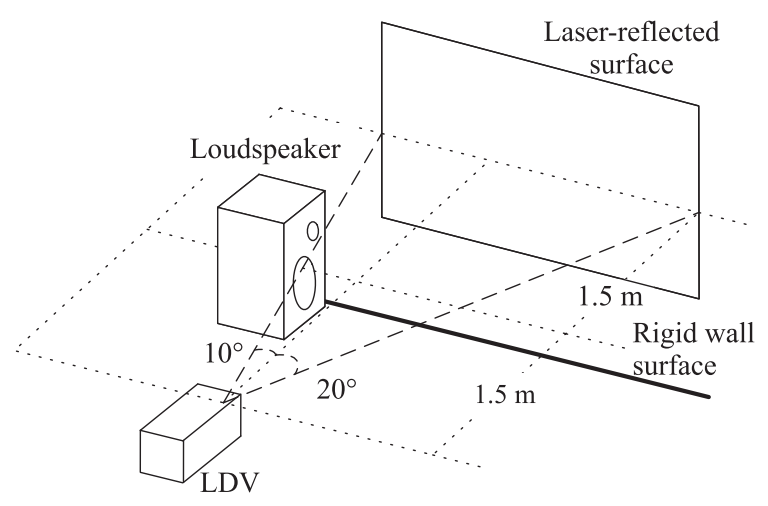

Fig. 2 Observation method for two-dimensional projection of sound.

\subsection{Experiment of Sound Field Observation with Two-dimensional Projection}

2.3.1. Observation of two-dimensional projection of a pulse sound wave

We conducted fundamental experiments using two types of loudspeaker. Figure 2 shows the arrangement of a loudspeaker and LDV. We use a measurement signal that contains one period of a $4 \mathrm{kHz}$ sine wave signal and silence long enough to reduce the reverberation of the room.

Figure 3 shows the result with a two-way loudspeaker (YAMAHA NS10M) and a power amplifier (YAMAHA $\mathrm{P} 4050)$. The sound pressure level is $110 \mathrm{~dB}$ at $1 \mathrm{~m}$ from the loudspeaker with a $4 \mathrm{kHz}$ sine signal. Similarly, Fig. 4 shows the result with a 2-by-4 array of flat-panel loudspeakers (WASEDA EE W-3232, $30 \mathrm{~cm} \times 30 \mathrm{~cm}$ ) connected in series.

Since the bandwidth division frequency of the 2-way loudspeaker is $2 \mathrm{kHz}$ ( $12 \mathrm{~dB} /$ oct attenuation), a spherical wave is mainly driven from a (dome-shaped) tweeter. We also observed another spherical wave driven from the (corn-shaped) woofer in Fig. 3. The input signal is one cycle of a sine signal. However, in Fig. 3, we observed more than one cycle of the signal and confirmed the damping effect of the loudspeaker and powered amplifier. On the other hand, as seen from Fig. 4, a flat wave was driven from the array of flat-panel loudspeakers. 

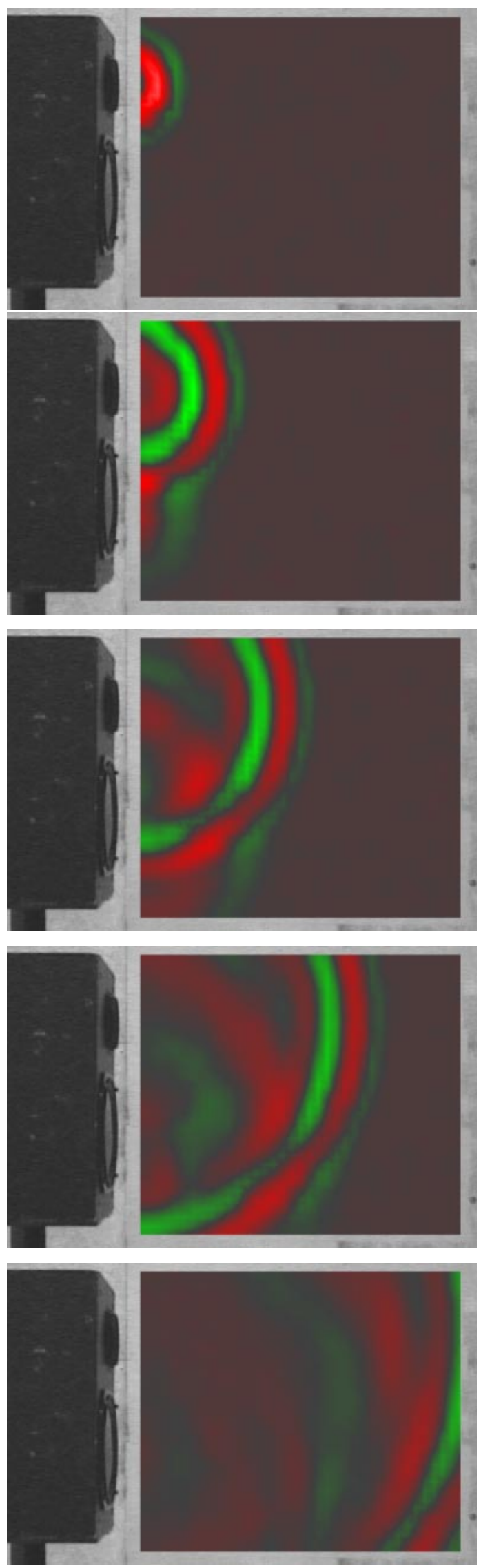

Fig. 3 Observation of pulse sound wave generated by 2 -way loudspeaker. Sound projections obtained in $0.3 \mathrm{~ms}, 0.6 \mathrm{~ms}, 0.9 \mathrm{~ms}, 1.2 \mathrm{~ms}$ and $1.8 \mathrm{~ms}$ in top-down order.

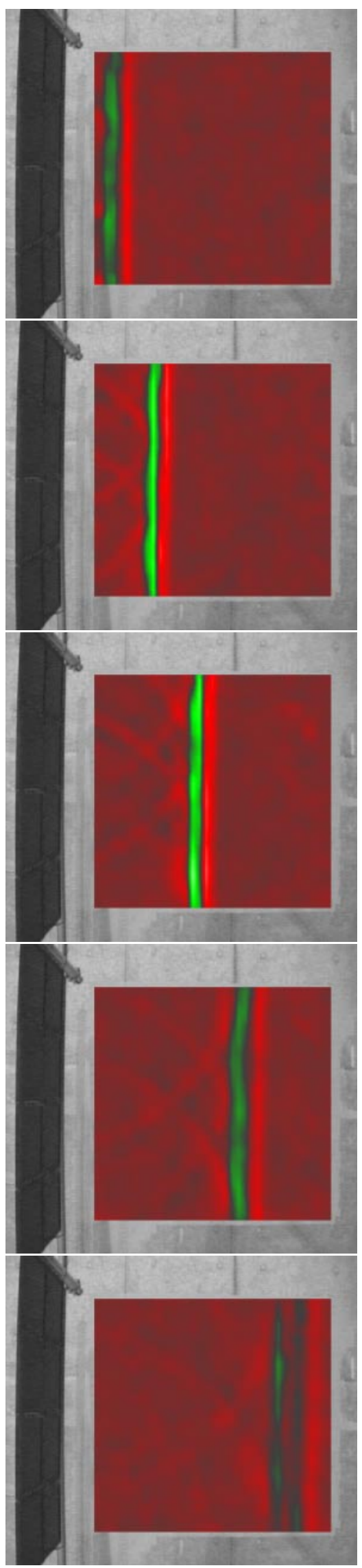

Fig. 4 Observation of pulse sound wave generated by flat-panel loudspeakers. Sound projections obtained in $1.0 \mathrm{~ms}, 1.5 \mathrm{~ms}, 2.0 \mathrm{~ms}, 2.5 \mathrm{~ms}$ and $3.0 \mathrm{~ms}$ in top-down order. 


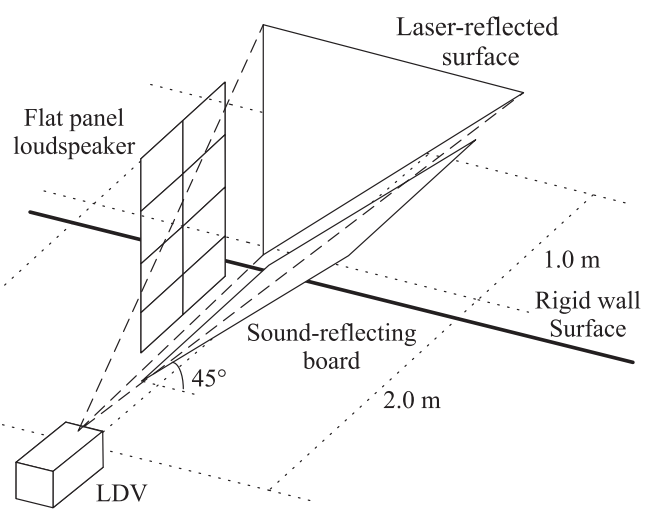

Fig. 5 Configuration for observation of reflection of pulse sound wave.

2.3.2. Observation of reflected sound wave with soundreflecting board

Next, we conduct an experiment with a two-dimensional laser projection to observe a reflection, off a soundreflecting board, of a pulse sound wave propagating from a flat-panel loudspeaker. Figure 5 shows an arrangement of flat-panel loudspeakers and the sound-reflecting board. One cycle of a $4 \mathrm{kHz}$ sine signal is used as the input pulse signal. The sound-reflecting board is a chip board with a thickness of $12 \mathrm{~mm}$ and a size of $1,820 \mathrm{~mm}$ by $910 \mathrm{~mm}$. The output sound pressure level is $100 \mathrm{~dB}$ at $1 \mathrm{~m}$ from the loudspeaker with the $4 \mathrm{kHz}$ sine signal. The air temperature in the experiment is 14 degrees.

Since the sound-reflecting board is tilted at a 45degrees angle, we observed in Fig. 6 that the flat sound wave propagated from the flat-panel loudspeaker is reflected, followed by a 90-degrees change in the direction of sound propagation. Then, we continue to observe that the sound wave reflected by the ceiling of the room is again reflected by the sound-reflecting board as this experiment is conducted in a closed space.

Note that the observed information is a projection of the sound field which is different from the cross-sectional surface of the sound field.

\section{RECONSTRUCTION OF SOUND FIELD INFORMATION OF PULSE SOUND WAVE WITH A LASER TOMOGRAPHY}

\subsection{Method of Reconstruction}

As shown in Sect. 2.1, the information obtained with a laser interferometer is integrated sound information on the laser path. In this section, we reconstruct information at the points by computed tomography.

Firstly, we briefly explain the reconstruction method. For reconstruction, the measured sound field requires projection information from every direction. Each set of measurement data taken from each direction using the laser interferometer is considered as a projection. Then, we

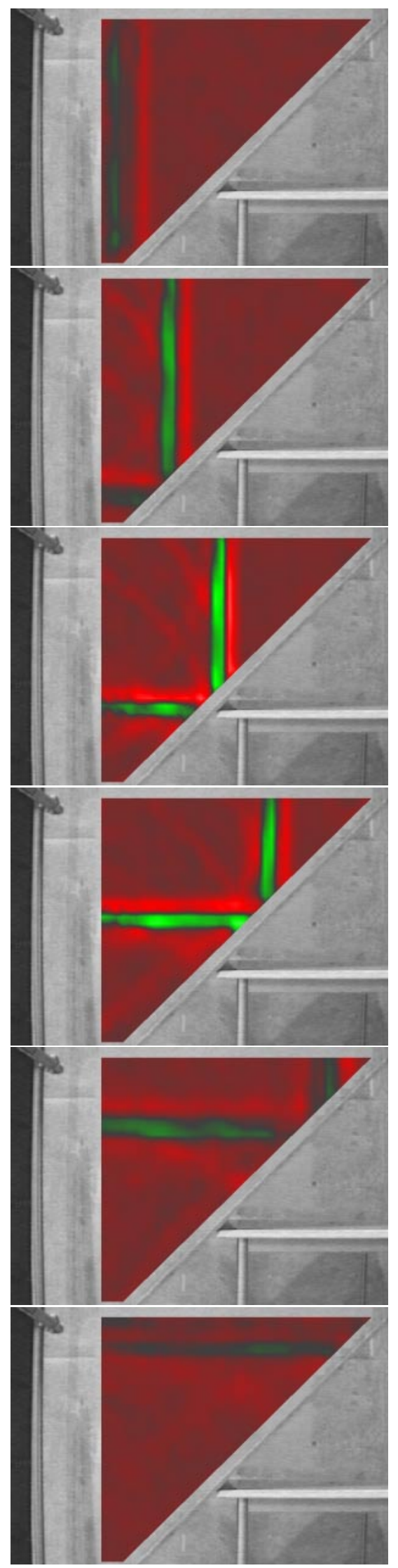

Fig. 6 Two-dimensional projection of reflection of pulse sound wave with laser interferometer. Sound projections obtained in $1.5 \mathrm{~ms}, 2.0 \mathrm{~ms}, 2.5 \mathrm{~ms} 3.0 \mathrm{~ms}$, $3.5 \mathrm{~ms}$ and $4.0 \mathrm{~ms}$ in top-down order. 


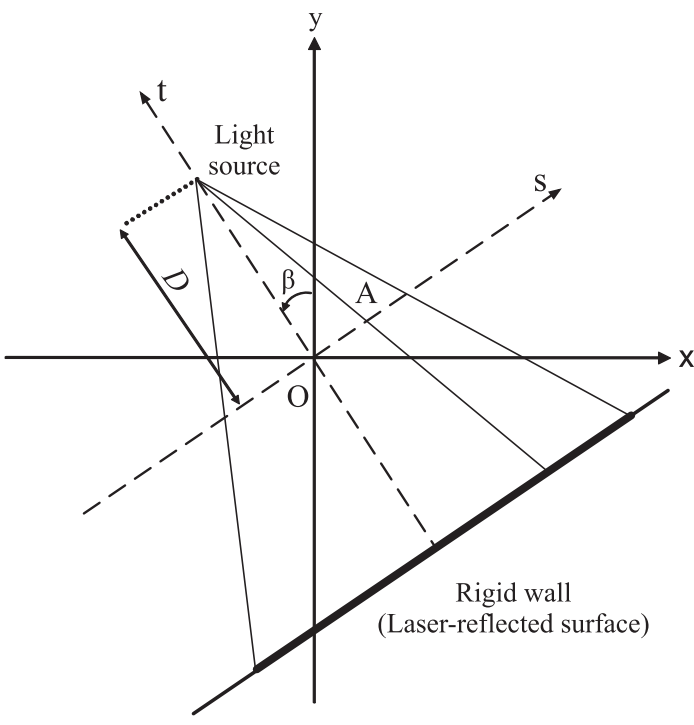

Fig. 7 Reconstruction method when reflected points are placed at the same line in even interval.

reconstruct the distribution of the temporal differentiation of refractive indexes, and the relationship between the refractive index and sound pressure is used to convert the former into the latter.

We use the convolution integral method for reconstruction [15]. Let the Cartesian coordinates in the plane be $(x, y)$, and the polar coordinates be $(r, \phi)$. We model the measured sound field after a distribution of temporal differentiation of the refractive indexes. We assume that the refractive indexes outside of the measured area are uniform and that the temporal differentiation of refractive indexes is zero. The measured distribution of the temporal differentiation of refractive indexes is represented by $f(x, y)$. The method of measuring the projection data is to rotate a laser source around the sound field with a scanning LDV sequentially. At each position of the laser source, we measured the projection data by scanning the laser at regular intervals on the laser reflection surface. The sound field to be measured is reproducible and can be moved and rotated in the same way as a direct sound from a loudspeaker. In this study, instead of rotating the measurement system, we rotate the sound field and obtain the projection data from every direction.

As shown in Fig. 7, the angle of rotation of the light source from axis $y$, the distance between the light source and rotation axis $O$, and the distance between the origin $O$ and intersection point $A$ of the light path that passes through the reconstructed points and axis $x$ rotated at an angle $\beta$ are represented by $\beta, D$ and $s$, respectively. The data of the projection that passes through point $A$ is represented by $R_{\beta}(s)$.

The distribution of the temporal differentiation of refractive indexes is obtained by

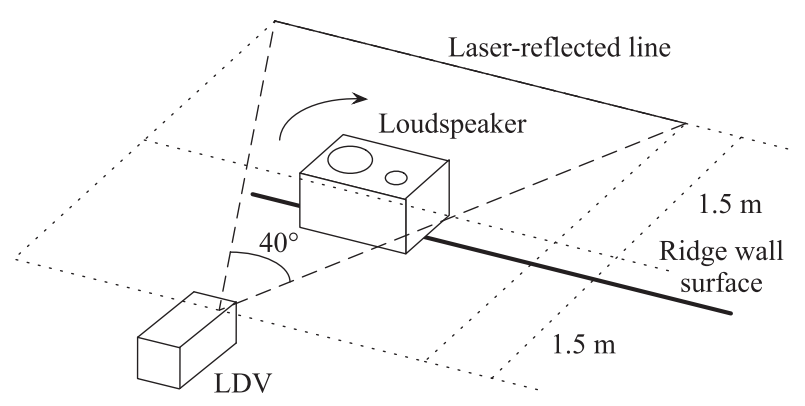

Fig. 8 Measurement method of projection for computed tomography.

$$
\begin{aligned}
& f(r, \phi)=\int_{0}^{2 \pi} \frac{1}{U^{2}} \int_{-\infty}^{\infty} R_{\beta}(s) g\left(s^{\prime}-s\right) \frac{D}{\sqrt{D^{2}+s^{2}}} d s d \beta, \\
& U(r, \phi, \beta)=\frac{D+r \sin (\beta-\phi)}{D}, \\
& g(s)=\frac{1}{2} h(s),
\end{aligned}
$$

where $h$ is the impulse response of a filter function to reduce high-frequency components, etc. [5]. $s^{\prime}$ corresponds to the value of $s$ when the light path passes through reconstructed point $(r, \phi)$. Since the measured projection data is discontinuous, $R_{\beta}\left(s^{\prime}\right)$ is calculated by a linear interpolation method. When we measure the projection data using the LDV, a laser passes through the measured space, is reflected by the ridge wall surface and returns with sufficiently high speed to ignore the changes in the sound field. Therefore, we use a half of the changes in the measured light path length as projection data.

\subsection{Experiment}

We conduct an experiment on the reconstruction of sound field information at each point for the direct sound generated by a 2-way loudspeaker system (YAMAHA NS$10 \mathrm{M}$ ) and a powered amplifier (YAMAHA P4050).

As shown in Fig. 8, we reconstruct the sound field information in the plane at the distance of $30 \mathrm{~cm}$ above the surface of the 2-way loudspeaker that is installed facing upward. The computed tomography method requires measurement from every direction around the loudspeaker. Since the object of measurement is the direct sound generated by the loudspeaker, we obtain the projection data by rotating the loudspeaker and not the measurement system.

The rotation axis is the center of reconstruction; the center is placed at one-third of the loudspeaker enclosure length from the tweeter-side edge. Therefore, the tweeter center is placed on the line passing through point $(0.07,0.03)$ in parallel with the rotation axis. Similarly, the center of the woofer is placed on the line passing through point $(-0.10,0)$. 
We use the NSK Megatorque motor system (EA32 Driver Unit, YS Motor) to rotate the loudspeaker. We use a level guide to ensure that the loudspeaker-rotating motor's top surface, the upward-facing loudspeaker's front and the LDV laser head's top surface are installed horizontally. Since the laser light is visible, we adjust the laser to pass through the rotation axis.

The loudspeaker's angle of rotation is 360 degrees in 15-degree increments; the number of measurement points for each projection is 101. As described in the previous sections, we use one cycle of a $4 \mathrm{kHz}$ sine signal as a measurement signal.

Figures 9-12 show distributions of the temporal differential of sound pressures calculated from the relationship between the refractive index and sound pressure [5]; the reconstruction of the distribution of refractive indexes is calculated from the differential of the light path length measured using the LDV. As shown in Figs. 9 and 10, the pulse sound wave propagates from the center of the tweeter. Figures 11 and 12 show the effect of the woofer. In addition, it is possible to convert the distributions to sound pressure distributions by the integration of the temporal differential.

\section{CONCLUSIONS}

We observed the traveling sound waves using a laser and a pulse sound wave. For example, we observed the reflection of a sound wave by the sound-reflecting board. We also showed that it is possible to reconstruct the information at each point of the measured area by the computed tomography method.

The observation of the sound wavefront is suitable for comparison with the results of computer simulation. In future research, we will apply our proposed method to the analysis of more complicated sound fields. Since the reconstructed information at each point is different from the commonly used physical quantity, we intend to convert it to a common physical quantity such as the sound pressure distribution.

\section{REFERENCES}

[1] Y. Yamasaki and T. Ito, "Measurement of spatial information in sound fields by closely located four point microphone method," J. Acoust. Soc. Jpn. (E), 10, 101-110 (1989).

[2] Y. Yamasaki, H. Tachibana, M. Morimoto, Y. Hirasawa and Z. Maekawa, "Survey on the acoustics of concert halls in European countries (The 2nd report): Measured results by four-point microphone method," J. Acoust. Soc. Jpn. (J), 43, 277-285 (1987) (in Japanese).

[3] N. Saito and Y. Yamasaki, "Sound recording without diaphragm," Proc. Spring Meet. Acoust. Soc. Jpn., pp. 479480 (2002) (in Japanese).

[4] T. Takizawa, N. Saito, T. Konishi and Y. Yamasaki, "Sound acpuisition without diaphragm in interspace using laser Doppler microphone," Proc. Spring Meet. Acoust. Soc. Jpn.,
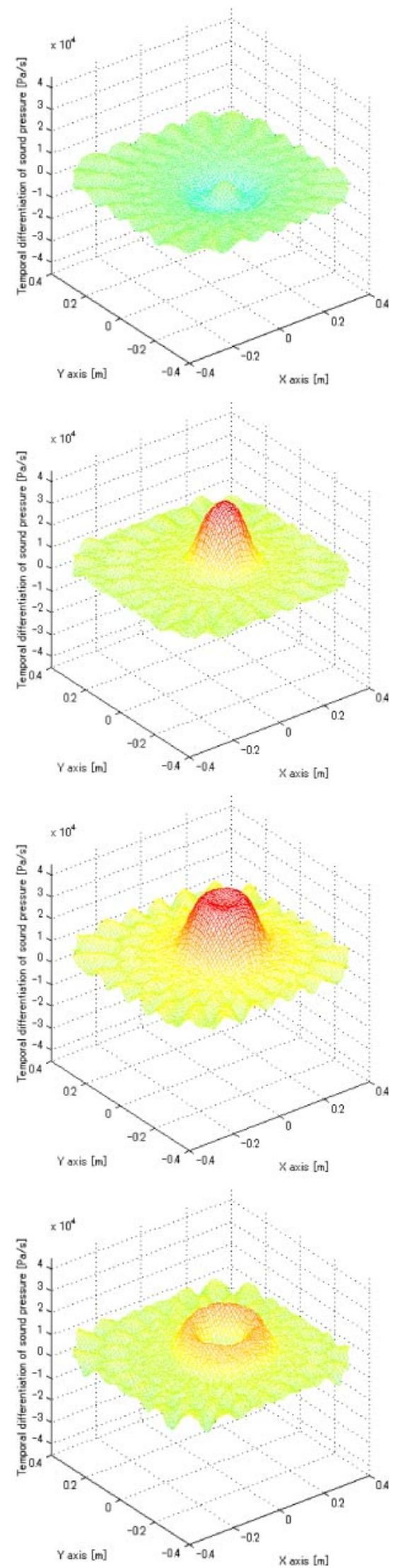

Fig. 9 Observation of the plane surface at $0.3 \mathrm{~m}$ distance from the front surface of the loudspeaker. Sound information reconstructed in $0.98 \mathrm{~ms}, 1.02 \mathrm{~ms}$, $1.05 \mathrm{~ms}$ and $1.09 \mathrm{~ms}$ in top-down order. 

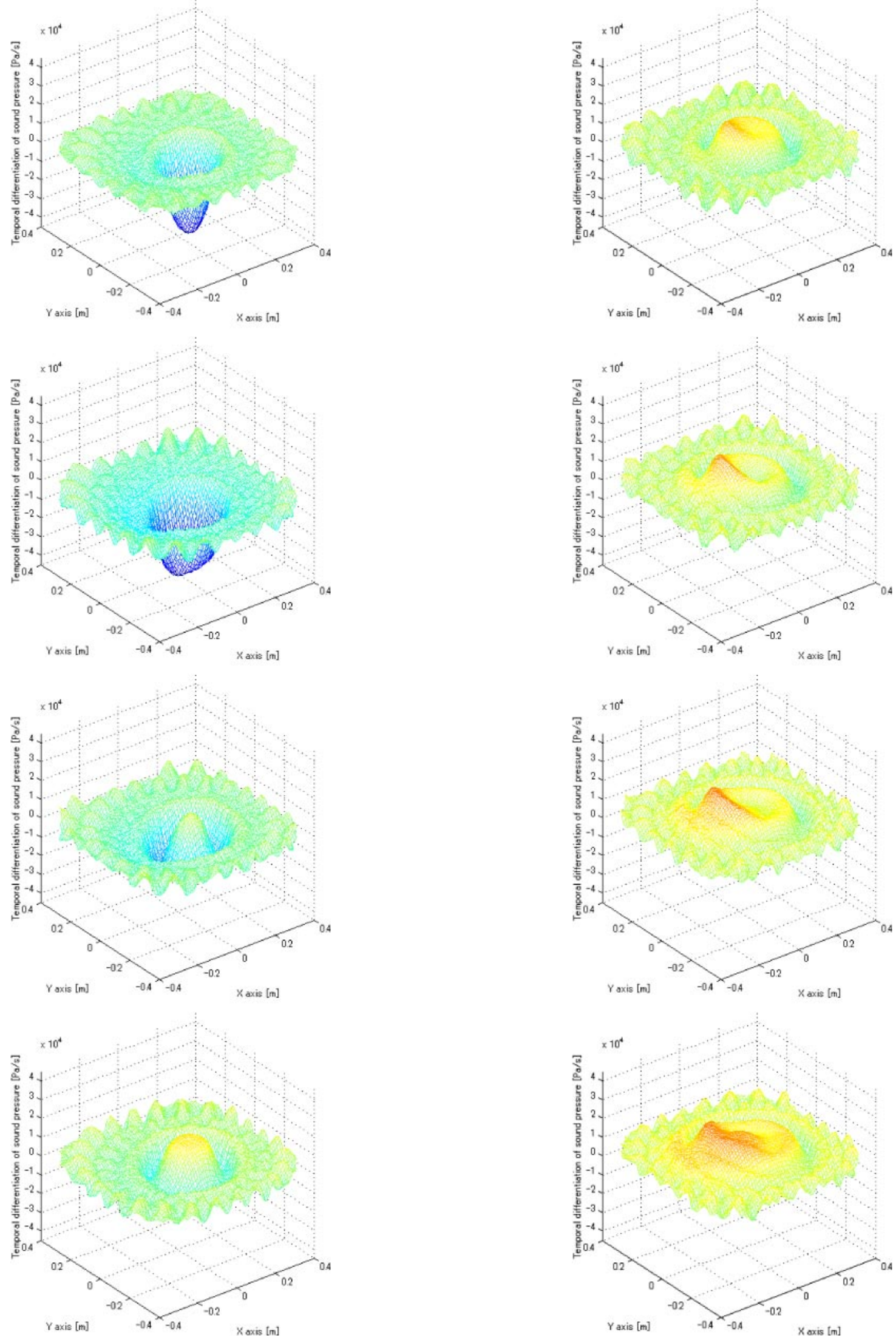

Fig. 10 Observation of the plane surface at $0.3 \mathrm{~m}$ distance from the front surface of the loudspeaker. Sound information reconstructed in $1.13 \mathrm{~ms}, 1.17 \mathrm{~ms}$, $1.21 \mathrm{~ms}$ and $1.25 \mathrm{~ms}$ in top-down order. 


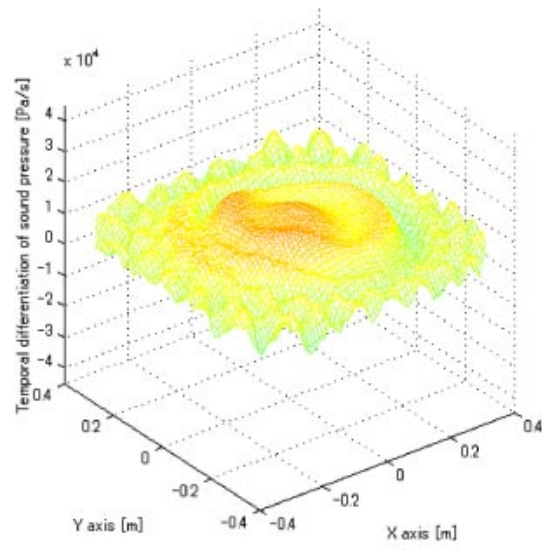

pp. 595-596 (2003) (in Japanese).

[5] Y. Ikeda, M. Goto, N. Okamoto, T. Takizawa, Y. Oikawa and Y. Yamasaki, "A measurement of reproducible sound field with laser computed tomography," J. Acoust. Soc. Jpn. (J), 62, 491-499 (2006) (in Japanese).

[6] G. W. Faris and R. L. Byer, "Three-dimensional beamdeflection optical tomography of a supersonic jet," Appl. Opt., 27, 5202-5212 (1988).

[7] H. M. Hertz, "Experimental determination of 2-D flame temperature fields by interferometric tomography," Opt. Commun., 54, 131-136 (1985).

[8] H. Kikuta, T. Nakano and K. Iwata, "Comparison of two-beam and differential interferometries for measurement of refractiveindex distributions by the optical CT technique," Trans. Jpn. Soc. Mech. Eng. C, 63, 190-196 (1997) (in Japanese).

[9] M. Yoshioka, K. Mizutani and K. Nagai, "Sonic field measurement using light computerized tomography," Jpn. J. Appl. Phys., 36, 3199-3202 (1997).

[10] K. Nakamura, "Measurements of high power ultrasound in the air through the modulation in the refractive index of air," IEICE Tech. Rep., US2001-9, pp. 15-20 (2001) (in Japanese).

[11] M. Hirayama, K. Nakamura and S. Ueha, "Directional sound field measurements by detecting the refractive index modulation of air," IEICE Tech. Rep., EA2002-122, Vol. 102, No. 607, pp. 13-18 (2003) (in Japanese).

[12] Y. Ikeda, M. Goto, T. Takizawa, Y. Oikawa and Y. Yamasaki, "3D Measurements of sound field based on reconstruction from laser projections," IEICE Tech. Rep., EA 104(497), pp. 43-48 (2004) (in Japanese).

[13] N. Okamoto, M. Goto, Y. Ikeda, T. Konishi, Y. Oikawa and Y. Yamasaki, "Application of reconstruction from laser projections to acoustic design," Proc. Spring Meet. Acoust. Soc. Jpn., pp. 819-820 (2005) (in Japanese).

[14] Y. Yamasaki, T. Konishi, Y. Oikawa, Y. Tokita and T. Fukuyama, "Acoustics of environmental information laboratories in Honjo campus," Proc. Autumn Meet. Acoust. Soc. Jpn., pp. 1079-1080 (2004) (in Japanese).

[15] A. C. Kak and M. Slaney, Principles of Computerized Tomographic Imaging (IEEE Press, New York, 1988).

Fig. 12 Observation of the plane surface at $0.3 \mathrm{~m}$ distance from the front surface of the loudspeaker. Sound information reconstructed in $1.45 \mathrm{~ms}, 1.48 \mathrm{~ms}$, $1.52 \mathrm{~ms}$ and $1.56 \mathrm{~ms}$ in top-down order. 